\title{
Efecto De La Fertilizacion Fosforo Azufrada Sobre El Sistema Radical Y El Rendimiento De Soja En El Centro De Argentina
}

\author{
Ing. Agr. MSc. M. D. Bongiovanni \\ Ing. Agr. MSc G. A. Cerioni \\ Ing. Agr. A. Gonzalez Giorgi \\ E. Dobla (Estudiante Avanzado) \\ Facultad de Agronomía y Veterinaria, \\ Universidad Nacional de Río Cuarto, Córdoba, Argentina
}

doi: 10.19044/esj.2016.v12n27p18 URL:http://dx.doi.org/10.19044/esj.2016.v12n27p18

\begin{abstract}
The objective was to evaluate grain the effect of sulfur and phosphorus fertilization on root growth, nodules and soybean yield. The test was performed at $25 \mathrm{~km}$ west of Coronel Baigorria department Río Cuarto; Córdoba, Argentina on a Haplustol entic from the series Alpa Corral. Treatments applied were made different doses of single superphosphate (21\% $\mathrm{P}_{2} 0_{5}$ y $12 \%$ de S).TO: control treatment, no fertilizerT1: 5,5 $\mathrm{Kg} \mathrm{ha}^{-1} \mathrm{de}$ P y 7,2 Kg ha- ${ }^{-1}$ de S; T2: 10,9 Kg ha-1e P y 14,4 Kg ha-1 de S y T3: 16,48 $\mathrm{Kg} \mathrm{ha}^{-1}$ de $\mathrm{P}$ y $21 \mathrm{Kg} \mathrm{ha}^{-1}$. During crop cycle the following observations and measurements: emerged seedlings, root dry weight, number of nodules, primary root diameter, root length density (DLR), and determine harvest, crop yield ( $\mathrm{kg} / \mathrm{ha})$ and levels of available soil phosphorus at harvest. In this research project the application of phosphorus-sulfur fertilizer produced changes in soybean root system, by increasing dry weight of roots, generating lateral growth, improving the symbiosis with nitrogen-fixing bacteria which result in more number of nodules per root. Fertilization also modified rooting patterns increasing DLR in most fertilizer treatments, effects that resulted in an increase in soybeans yield.
\end{abstract}

Keywords: Roots, Glicine max (L) Merr., Fertilization, Phosphorus

\section{Resumen}

El objetivo de la experiencia fue evaluar el efecto de la fertilización fosforada y azufrada sobre el crecimiento de raíces, nodulación y rendimiento de un cultivo de soja. El ensayo se realizó a $25 \mathrm{~km}$ al oeste de la 
localidad de Coronel Baigorria, departamento Río Cuarto; Córdoba, Argentina sobre un Haplustol éntico perteneciente a la serie Alpa Corral. Los tratamientos realizados fueron diferentes dosis de superfosfato simple $(21 \%$ P205 y 12\% de S). El T0: Tratamiento testigo, sin fertilizante; T1: 5,5 Kg ha-1 de $\mathrm{P}$ y 7,2 Kg ha-1 de S; T2: $10,9 \mathrm{Kg}$ ha-1 de $\mathrm{P}$ y 14,4 Kg ha-1 de S y T3: $16,48 \mathrm{Kg}$ ha-1 de $\mathrm{P}$ y $21 \mathrm{Kg}$ ha-1. Durante el ciclo del cultivo se realizaron las siguientes observaciones y mediciones: número de plantas emergidas, peso seco de las raíces, cantidad de nódulos, diámetro de la raíz primaria, densidad de longitud de raíces (DLR) y a cosecha se determinó el rendimiento de granos por superficie (kg/ha) y los niveles de $\mathrm{P}$ disponible en el suelo luego de la cosecha del cultivo. Para la situación en estudio la aplicación de fertilizantes fosfo-azufrados produjo cambios en el sistema radical de la soja, aumentando el peso seco de las raíces y el crecimiento lateral de las mismas, favoreciendo la simbiosis con bacterias fijadoras de nitrógeno, dando como resultado una mayor cantidad de nódulos por raíces. También la fertilización modificó los patrones de enraizamiento, aumentando la DLR en los tratamientos con más fertilizantes, efectos que se tradujeron en un aumento en la producción de granos de soja.

Palabras clave: Roots, Glicine $\max (\mathrm{L})$ Merr., Fertilization, Phosphorus

\section{Introducción}

La provincia de Córdoba cuenta con una superficie agrícola total de 8.337.125 has, de las cuales un $58 \%$ son sembradas con soja (MAGyA, 2014), dentro de este porcentaje se encuentran incluidas las 510.000 has sembradas en el departamento Rio Cuarto de las cuales se producen rindes promedios entre 26 y 29 quintales por hectáreas (MAGyA, 2014).

El cultivo de soja (Glycine max (L.) Merr.) es uno de los más extractivos puesto que devuelve muy pocos nutrientes al suelo (Gutierrez Boem y Scheiner, 2007). En condiciones mejoradas de manejo del cultivo, se observan rendimientos que llegan a duplicar los promedios regionales, siendo el manejo integral de la nutrición y las rotaciones, algunos de los elementos que con mayor frecuencia contribuyen al mejoramiento en el rendimiento de la soja (Díaz-Zorita et al., 2003). Ante deficiencias marcadas de $\mathrm{P}$ un incremento en la provisión de este, aumenta el peso seco de raíces y en particular la longitud de las raíces (Lynch et al. 2012).

La fijación biológica de nitrógeno requiere del aporte de recursos energéticos y de nutrientes específicos (Gan et al., 2002). Las deficiencias de fósforo $(\mathrm{P})$ reducen la nodulación, la cantidad de $\mathrm{N}$ fijado y la producción de granos (Tsvetkova \& Georgiev, 2003, Fontaneto, 2004). El requerimiento de nutrientes podrá ser total o parcialmente suplido por el suelo, cuando los valores del mismo superen los niveles críticos para ese nutriente, como para 
el caso del $\mathrm{P}$ se logran un rendimiento relativo del 90-95\% con niveles de 8 $12 \mathrm{mg} \mathrm{kg}^{-1}$ en el suelo (Berardo, 2003).

Los niveles de $\mathrm{P}$ disponible en el sur de Córdoba, Argentina difirieren significativamente entre departamentos siendo los valores medios encontrados en el departamento Río Cuarto de 11,3 ppm (Bongiovanni et al., 2010). Sin embargo, a pesar de los bajos niveles de $\mathrm{P}$ ya mencionada $\mathrm{y}$ de los niveles de extracción de nutrientes del cultivo, los nutrientes repuestos por fertilización son sólo una pequeña proporción de lo que se exporta.

Melgar et al. (1995), sostienen que el valor de $10 \mathrm{ppm}$ de fósforo extractable puede ser considerado como umbral crítico para decidir la fertilización en soja. En un análisis de 65 ensayos de fertilización con $\mathrm{P}$ realizado por este autor detectó incrementos de 355, 214, y $34 \mathrm{~kg}$ ha-1 cuando se fertilizó la soja en suelos con niveles inferiores a 9 ppm, de 10 a 14 ppm y mayores de 15 ppm, respectivamente.

Díaz Zorita et al. (2010) en suelos vertisoles encontraron respuesta significativa en rendimiento de soja para diferentes niveles de fertilización fosforada, no encontrando diferencias significativas en el número y biomasa de nódulos en raíces primarias y laterales.

Cuando se tratan de mejorar niveles nutricionales con la aplicación de fertilizantes, es importante tener en cuenta la forma de aplicación ya que la soja es un cultivo proclive a sufrir efectos fitotóxicos por la aplicación de fertilizantes en la línea de siembra, afectando directamente el número de plantas emergidas (Ferraris et al., 2004).

El objetivo de la experiencia fue evaluar el efecto de la fertilización fosforada $\mathrm{y}$ azufrada sobre el crecimiento de raíces, nodulación $\mathrm{y}$ rendimiento de un cultivo de soja.

\section{Materiales y métodos}

El ensayo se realizó en un establecimiento agrícola ubicado a $25 \mathrm{~km}$ al oeste de la localidad de Coronel Baigorria, departamento Río Cuarto;

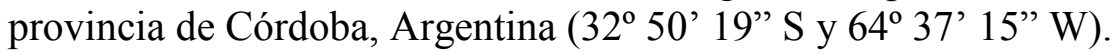

El suelo donde se llevó a cabo el ensayo se encuadra dentro de la clasificación taxonómica Haplustol éntico perteneciente a la serie Alpa Corral, que se caracteriza por ser profundo, bien drenado, con desarrollo a partir de materiales loéssicos franco limoso y se vincula a relieves de lomas onduladas, con una historia agrícola de rotaciones maíz-soja.

Previo a la siembra se realizó un análisis de suelo de los primeros 20 cm y se determinó: $1,48 \%$ de materia orgánica por Walkley-Black (Nelson and Sommer, 1982) 7,9 ppm de N-NO3-, 4,9 ppm de Fósforo disponible por Bray and Kurtz I (Olsen and Sommers, 1982) y 6,4 de pH.

Se utilizó el cultivar de soja DM50048 de hábito de crecimiento indeterminado, en surcos a $42 \mathrm{~cm}$ entre hileras a una densidad de 18 semillas 
por $\mathrm{m}$, previo a la siembra fue tratada con fungicida e inoculada con Bradirizobyum japonicum.

El diseño utilizado fue en bloques completos al azar, con tres repeticiones. Los tratamientos realizados fueron diferentes dosis de Superfosfato simple (21\% $\mathrm{P}_{2} \mathrm{O}_{5}$ y $12 \%$ de $\left.\mathrm{S}\right)$. El T0: Tratamiento testigo, sin fertilizante; T1: 5,5 $\mathrm{Kg} \mathrm{ha}^{-1}$ de $\mathrm{P}$ y 7,2 $\mathrm{Kg} \mathrm{ha}^{-1}$ de $\mathrm{S}$; T2: 10,9 $\mathrm{Kg} \mathrm{ha}^{-1}$ de P y 14,4 $\mathrm{Kg} \mathrm{ha}^{-1}$ de S y T3: 16,48 $\mathrm{Kg} \mathrm{ha}^{-1}$ de P y $21 \mathrm{Kg} \mathrm{ha}^{-1}$.

Las precipitaciones totales acumuladas durante el periodo Noviembre - Abril fueron de 959,5.

Durante el ciclo del cultivo se realizaron las siguientes observaciones y mediciones:

Establecimiento del cultivo: se contabilizó el número de plantas emergidas a los 20 DDS, para ello se contaron las plantas en $10 \mathrm{~m}$ de surco por tratamiento y repetición.

Crecimiento de raíces y nodulación: en la etapa fenológica de madurez se extrajeron 3 plantas contiguas con su correspondiente pan de tierra de 0,30 $\mathrm{m}$ de ancho por 0,20 $\mathrm{m}$ de profundidad, en cada parcela con seis repeticiones (Figura 1). Posteriormente en laboratorio se procedió al lavado con agua a presión, separando la tierra de las raíces, de modo de poder determinar los siguientes parámetros: Peso seco de las raíces, cantidad de nódulos totales registrando su ubicación sobre raíces primarias y secundarias, número de raíces secundarias por planta y diámetro de la raíz primaria.

Densidad de longitud de raíces (DLR): en la etapa fenológica R4-R5 se realizaron calicatas en cada tratamiento, de $190 \mathrm{~cm}$ de ancho por $100 \mathrm{~cm}$ de largo, por $130 \mathrm{~cm}$ de profundidad. En ella se cartografió y se contaron las raíces con la ayuda de una grilla metálica (Bhom, 1979) con modificaciones, haciendo coincidir el centro de la grilla con alguna línea de siembra, y de este modo se abarcaron tres líneas simultáneamente (Figura 2). Los datos obtenidos fueron utilizados para el cálculo de la DLR.

A madurez se determinó el rendimiento de granos por superficie $\left(\mathrm{kg} . \mathrm{ha}^{-1}\right)$ y los niveles de $\mathrm{P}$ disponible en el suelo luego de la cosecha del cultivo.

Los resultados obtenidos fueron procesados mediante ANOVA y la comparación de medias con el test de Duncan $(p=0,05)$ utilizando el programa estadístico INFOSTAT (Di Renzo et al., 2011).

\section{Resultados y discusión}

Establecimiento del cultivo: En concordancia con lo planteado por Ferraris (2004) y Ventimiglia (2006), la aplicación de fertilizante en la línea de siembra modificó el número de plantas establecidas $(\mathrm{p}<0,05)$, T0 tuvo mayor número de plantas por superficie $(34 \mathrm{pl} \mathrm{m} 2)$ respecto a los 
tratamientos con fertilizantes que presentaron una reducción promedio de $17 \%$ menos plantas por superficie. Por su parte los tratamientos fertilizados (T1, T2 y T3) no mostraron diferencias significativas entre sí con valores de 29; 28 y $27 \mathrm{pl} \mathrm{m}^{-2}$ respectivamente.

Crecimiento de raíces y Nodulación: Los parámetros evaluados en las raíces de las plantas de soja sufrieron modificaciones con la aplicación de (Tabla $\mathrm{N}^{\mathrm{o}}$ 1). El peso seco de raíces por planta presentó diferencias significativas entre los tratamientos de fertilización y el testigo. Comparando los tratamientos fertilizados $\mathrm{T} 2$ fue el que tuvo mayor peso seco de raíces con respecto de los tratamientos, $\mathrm{T} 1$ y T3. El número de raíces laterales por planta para los distintos tratamientos de fertilización presentaron diferencias significativas $(\mathrm{p}<0,05)$ en concordancia con lo planteado por Lynch et al. (2012) que encontraron mayor desarrollo de raíces por efecto del fósforo en leguminosas anuales. Los tratamientos con mayor nivel de fertilización T2 y T3 tuvieron mayor cantidad de raíces sin diferencias significativas entre sí. Por su parte T1 y T0, presentaron los menores valores sin diferencias significativas entre ellos. El diámetro medio de raíz principal de $0-10 \mathrm{~cm}$ aumentó con la fertilización (Tabla $\mathrm{N}^{\mathrm{o}}$ 1). T2 y T3 fueron estadísticamente superiores a T0.

Número de nódulos por raíz: El número de nódulos ubicados sobre la raíz principal, aumentó en el tratamiento T2 en un $52 \%$ más de nódulos respecto a T0 (Tabla $N^{\circ} 1$ ). Por su parte T1 y T3 fueron levemente mayores que T0 sin diferencias significativas entre ellos. El número de nódulos ubicados sobre las raíces laterales de la planta se modificaron con la fertilización, T2, tuvo un $70 \%$ más de nódulos que el tratamiento sin fertilizar T0 y que T1, sin diferir estadísticamente de T3. Respecto al número de nódulos totales por planta, T2 tuvo un $62 \%$ más de nódulos que el testigo T0 y $25 \%$ superior a T1, aunque sin diferir estadísticamente de T3. Esto concuerda con los resultados de Fontanetto et al. (2004) y Díaz Zorita et al. (2010) quienes describieron aumentos en el número y en la biomasa de nódulos al incrementarse los aportes de $\mathrm{P}$ sugiriendo que en las condiciones del estudio que realizaron el $\mathrm{P}$ era limitante para la normal formación de nódulos y su crecimiento, condiciones similares al presente estudio donde los niveles de $\mathrm{P}$ están muy por debajo de los niveles críticos citados por Berardo (2003). Según Miao et al.,(2007), al incrementar los aportes de P se incrementa la biomasa de nódulos porque la deficiencia de $\mathrm{P}$ inhibe el desarrollo de los mismos. De acuerdo con lo planteado por Tsvetkova \& Georgiev (2003), en el presente estudio la relación entre los niveles de $\mathrm{P}$ y el número de nódulos totales por planta fue muy estrecha y significativa $(\mathrm{R} 2=0,994, \mathrm{p}<0,0001)$ y ajustando al modelo lineal: $\mathrm{y}=1.882 \mathrm{x}+47.7$ (Figura $\mathrm{N}^{\mathrm{o}} 3$ ). Estos datos concuerdan con lo deducido por los autores antes mencionado quienes también encontraron una correlación positiva entre 
ambas variables. La funcionalidad y efectividad de la fijación biológica requiere del aporte de recursos energéticos y de nutrientes específicos (Panzieri et al., 2000). Entre estos, las deficiencias de fósforo (P) reducen la nodulación, la cantidad de $\mathrm{N}$ fijado y la producción de granos (Tsvetkova \& Georgiev, 2003). Además, la oferta de $\mathrm{P}$ afecta indirectamente la fotosíntesis y así la disponibilidad de fotosintatos para ser traslocados a los nódulos (Christiansen y Graham, 2002).

Perfil de densidad de longitud de raíces: La DLR promedio al metro de profundidad en cada tratamiento fue, T0: 14,0; T1: 19,5; T2: 25,5 y T3: $21,0 \mathrm{~cm} \mathrm{~cm}^{-3}$. Por su parte T2 mostró el mayor valor de DLR con un $86,3 \%$ más que $\mathrm{T} 0$, los siguen $\mathrm{T} 1$ y $\mathrm{T} 3$ con 44,2 y $56,9 \%$ más que $\mathrm{T} 0$ respectivamente (Figura $\mathrm{N}^{\circ} 4$ ).

En la Figura $\mathrm{N}^{0} 5$ se muestra la DLR de los tratamientos para distintas profundidades. En los primeros centímetros del perfil se encontraron los mayores desarrollos de raíces, siendo los tratamientos fertilizados los de mayor DLR. El mayor desarrollo superficial encontrado es debido a que el sistema radical de la soja consiste de raíces laterales que surgen de los primeros $10-15 \mathrm{~cm}$ de la raíz primaria. Para la capa de 0 a $12 \mathrm{~cm}$ de profundidad existe una clara diferencia de valores promedios a favor de los T2, con un $67 \%$ más de DLR que T0. Por su parte T1 y T3 también se diferenciaron de T0 presentando un 49 y $41 \%$ más de DLR respectivamente. En estos últimos dos tratamientos se puede observar una leve diferencia a favor de T1, esto pudo deberse a la diferencia de número de plantas a favor de este último y a un posible efecto fitotóxico producido por cantidades elevadas de fertilizante en la misma línea de siembra que redujo en mayor medida el número de plantas y por ende la DLR de T3. En la capa que va desde los $12 \mathrm{~cm}$ de profundidad hasta $\operatorname{los} 24 \mathrm{~cm}$, en lo que respecta a valores promedios de DLR se destacan T3 y T2 con 74 y $58 \%$ más de DLR que T0. En la medida que analizamos capas más profundas, podemos observar una clara tendencia a que los mayores valores promedios de DLR los presenta $\mathrm{T} 2$, que se diferencian claramente con $\mathrm{T} 0$ en cuanto a valores promedios, tendencia que no se observa en todos los casos con respecto a los otros dos tratamientos (T1 y T3). La profundidad máxima alcanzada por las raíces no se evaluó en esta experiencia, solo se midió hasta los $109 \mathrm{~cm}$ de profundidad.

Del análisis de DLR surge que el \% de suelo ocupado por raíces fue siempre mayor en todos los tratamientos que llevaron alguna dosis de fertilizante (T1, T2, T3) con respecto a T0 (Figura $\mathrm{N}^{\mathrm{o}}$ 6).

Rendimiento: La Figura $\mathrm{N}^{0} 7$ muestra el rendimiento $(\mathrm{kg} / \mathrm{ha})$ del cultivo de soja bajo diferentes tratamientos con fertilizante fosforo azufrado. Los tratamientos con mayor cantidad de fertilizante aplicado (T2 y T3) tuvieron mayor rendimiento con valores de 2961 y $2960 \mathrm{~kg} / \mathrm{ha}$ sin diferencias estadísticas entre ellos. T0 y $\mathrm{T} 1$ fueron menores estadísticamente 
respecto a los anteriores (2329 y $2485 \mathrm{~kg} / \mathrm{ha}$ respectivamente) sin diferir entre ellos.

Esta respuesta del rendimiento a la aplicación de fertilizante coincide con lo descripto por Gutiérrez Boem y Thomas (2001), quienes afirman que el rendimiento del cultivo de soja, como el de otros cultivos puede descomponerse en número de granos y peso individual de los granos, pero aclaran que la caída en los rendimientos debido a una deficiencia de $\mathrm{P}$, se debe en general a una disminución en el número de granos. Una deficiencia fosforada en soja puede provocar una caída en los rendimientos por su efecto sobre la formación del área foliar $\mathrm{y}$, por lo tanto sobre la cantidad de radiación capturada, y también por su efecto sobre la eficiencia de conversión de esta radiación en materia seca. La suma de estos efectos provoca un menor crecimiento entre floración y comienzo de llenado de los granos, un menor número de granos $\mathrm{y}$, por lo tanto, un menor rendimiento. Se puede decir que hay cada vez más evidencia que la fertilidad fosforada de los suelos en la región pampeana es en muchos casos insuficiente para obtener buenos rendimientos.

La aplicación de fertilizante en la siembra dio como resultado un aumento del $\mathrm{P}$ en el suelo en la línea de siembra (al final del ciclo del cultivo), pasando de niveles de 4,8 ppm en T0 a valores de 5,8 ppm en T1, 7,5 ppm T2 y 13,5 ppm T3.

\section{Conclusion}

Para la situación en estudio la aplicación de fertilizantes fosfoazufrados produjo cambios en el sistema radical de la soja, aumentando el peso seco de las raíces y el crecimiento lateral de las mismas, favoreciendo la simbiosis con bacterias fijadoras de nitrógeno, dando como resultado una mayor cantidad de nódulos por raíces. También la fertilización modificó los patrones de enraizamiento, dando más DLR en los tratamientos con más fertilizantes, efectos que se tradujeron en un aumento en la producción de granos de soja.

\section{References:}

Berardo; A. 2003. Manejo del Fósforo en los sistemas de producción pampeanos. En: Simposio "El Fósforo en la Agricultura". pp 38-44. INPOFOS Cono Sur, Rosario

Bhom, 1979. Methods of Studying Root Systems. Ecological Studies 33. Springer, Berlin, Heidelberg, New York

Bongiovanni M. D.; Marzari R. y M. Ron 2010. Fósforo disponible en suelos agrícolas del sur de Córdoba y Sudeste de San Luis. XXII Congreso Argentino de la Ciencia del suelo, Rosario. Actas p121. 
Christiansen, I \& PH Graham. 2002. Variation in di-nitrogen fixation among Andean bean (Phaseolus vulgaris L.) genotypes grown at low and high levels of phosphorus supply. Field Crops Res. 73: 133-142.

Di Rienzo J.A., Casanoves F., Balzarini M.G., Gonzalez L., Tablada M., Robledo C.W. InfoStat versión 2011. Grupo InfoStat, FCA, Universidad Nacional de Córdoba, Argentina. URL http://www.infostat.com.ar

Díaz Zorita et al. 2010, M; Aranguren, J; Aguilar, R y Satorre, E. 2010. Fertilización fosfatada e inoculación de soja en vertisoles. Cienc. suelo, vol.28, n.2 pp. $215-222$

Díaz-Zorita, M. 2003. Soja: Criterios para el manejo de la fertilización del cultivo, p. 80-91. En Satorre, E. (ed.), El Libro de la Soja. SEMA- Servicios y Marketing, Buenos Aires. Argentina.

Ferraris, G., N. González, D. Bocanegra y A. Rivoltella. 2004. Eficiencia y fitotoxicidad de fuentes de fertilizantes fosforados en soja. Soja, Resultados de Unidades demostrativas del Proyecto Regional Agrícola. Desarrollo Rural EEA INTA Pergamino y General Villegas: 53-62.

Fontaneto H. Diaz Zorita M. y H. Vivas. 2004. Efecto de la inoculación y fertilización con fósforo y azufre sobre la nodulación y los rendimientos de soja. XIX Congreso Argentino de la Ciencia del Suelo. Paraná, 22 al 25 de junio de 2004. Resúmenes: 143.

Gan, Y; I Stulen; H Van Keulen \& PJC Kuiper. 2002. Physiological changes in soybean (Glycine max.) in response to $N$ and $P$ nutrition. Ann. Appl. Biol. 140: 319-329.

Gutierrez Boem F. H. y G. Thomas 2001. Leaf area development in soybean as affected by phosphorus nutrition and water deficits. Journal Plant Nutrition. 24(11):1711-1729.

Gutierrez Boem F. H y J. D Scheiner. 2007 Soja En: Fertilidad de Suelos y Fertilización de Cultivos. H.E. Echeverría y F.O. García (eds.). Ediciones INTA. Buenos Aires. Argentina, pp. 283-300

Lynch J., Marschner P. and Z. Rengel (2012) Effect of Internal and External Factors on Root Growth and Development in Marschner's Mineral Nutrition of Higher Plants. DOI:

Elsevier Ltd.pp 337-338.

MAGyP 2014 Información Agropecuaria provincial y departamental de la provincia de Córdoba en Estimaciones agrícolas

En:

http://magya.cba.gov.ar/upload/Estimaciones_CG_28_de_julio_de_2014.pdf Miao, SJ; YF Qiao; XZ Han \& MD An. 2007. Nodule formation and development in soybeans (Glycine max L.) in response to phosphorus supply in solution culture. Pedosphere 17: 36-43.

Nelson D. W. and Sommers L. E. (1982) Total Carbon, Organic Carbon and Organic Matter in . In: Page, A. L., Miller R. H. and Keeney D. R. (Editors) 
Methods of soil analysis, part 2. Chemical and Microbiological Properties. Agronomy Monograph 9. 2nd Edition, American Society of Agronomy and Soil Science Society of America, Madison, WI pp 539-577.

Olsen, S. R. and Sommers, L. E. (1982) Phosphorus. In: Page, A. L., Miller R. H. and Keeney D. R. (Editors) Methods of soil analysis, part 2. Chemical and Microbiological Properties. Agronomy Monograph 9. 2nd Edition, American Society of Agronomy and Soil Science Society of America, Madison, WI: 403-430.

PanzieriI, M; Marchettini N. \& TG Hallan. 2000. Importance of the Bradhyrizobium japonicum symbiosis for the sustentability of a soybean cultivation. Ecol. Modell.135: 301-310.v.

Tsvetkova, GE \& GI Georgiev. 2003. Effect of phosphorus nutrition on the nodulation, nitrogen fixation and nutrient use efficiency of Bradyrhizobium japonicum-soybean (Glycine max L. Merr.) symbiosis. Bulg. J. Plant. Physiol. Special Issue: 331-335.

Yahiya, M; Samiullah \& A Fatma. 1995. Influence of phosphorus on nitrogen fixation in chickpea cultivars. J. Plant Nutr.18: 719-727.

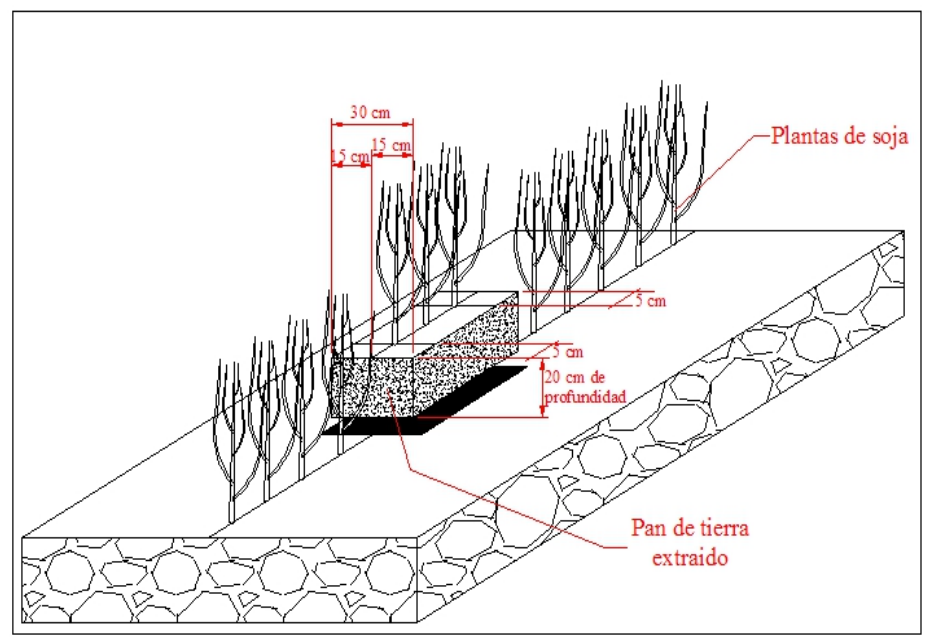

Figura 1. Diagrama de la extracción del pan de tierra para la determinación de crecimiento de raíces y nodulación de plantas de soja. 


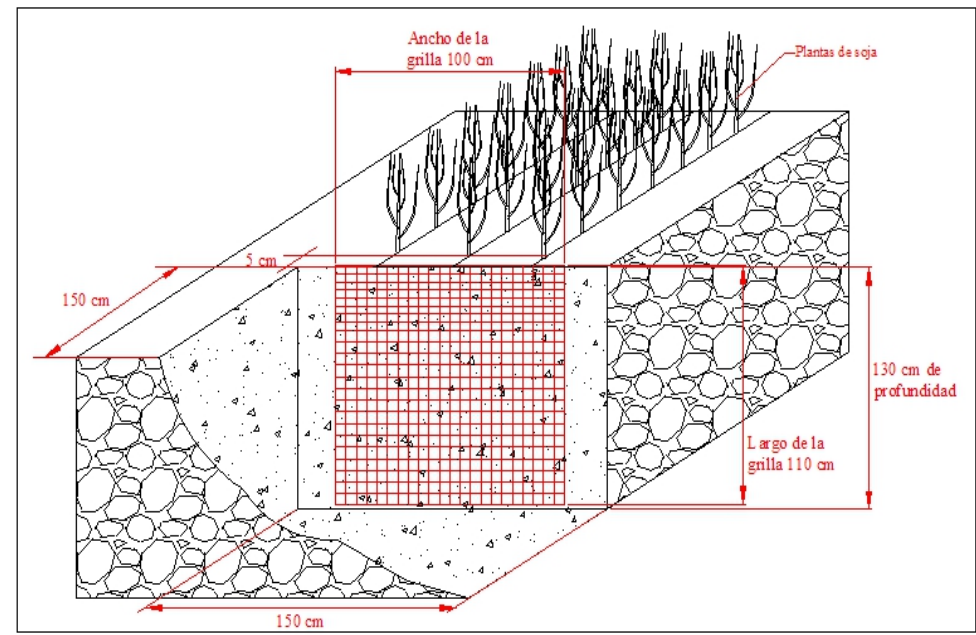

Figura 2. Diagrama de ubicación de la grilla metálica en la calicata para el cartografiado y determinación de DLR en raíces de soja

Tabla 1 Peso seco de raíces (g), número de raíces laterales, diámetro promedio de raíz de 0 $10 \mathrm{~cm}$ de profundidad, número de nódulos en raíz principal y lateral y nódulos totales.

\begin{tabular}{ccccccc}
\hline Tratamiento & $\begin{array}{c}\text { Peso } \\
\text { seco } \\
\text { raíces }(\mathrm{g} \\
\left.\text { planta }^{-1}\right)\end{array}$ & $\begin{array}{c}\text { Diámetro } \\
\text { de raíz } \\
\text { principal } \\
(\mathrm{cm})\end{array}$ & $\begin{array}{c}\mathrm{N}^{\mathrm{o}} \mathrm{de} \\
\text { raíces } \\
\text { laterales }\end{array}$ & $\begin{array}{c}\mathrm{N}^{\mathrm{o}} \\
\text { nódulos } \\
\text { raíz } \\
\text { principal }\end{array}$ & $\begin{array}{c}\mathrm{N}^{\mathrm{o}} \\
\text { nódulos } \\
\text { raíz } \\
\text { lateral }\end{array}$ & $\begin{array}{c}\mathrm{N}^{\mathrm{o}} \\
\text { nódulos } \\
\text { totales }\end{array}$ \\
\hline $\mathrm{T} 0$ & $3,87 \mathrm{a}$ & $0,41 \mathrm{a}$ & $40,17 \mathrm{a}$ & $10,5 \mathrm{a}$ & $36,4 \mathrm{a}$ & $46,9 \mathrm{a}$ \\
$\mathrm{T} 1$ & $5,33 \mathrm{~b}$ & $0,47 \mathrm{ab}$ & $41,89 \mathrm{a}$ & $11,6 \mathrm{a}$ & $46,9 \mathrm{ab}$ & $58,6 \mathrm{~b}$ \\
$\mathrm{~T} 2$ & $6,33 \mathrm{c}$ & $0,52 \mathrm{~b}$ & $48,22 \mathrm{~b}$ & $15,9 \mathrm{~b}$ & $61,8 \mathrm{c}$ & $77,7 \mathrm{c}$ \\
$\mathrm{T} 3$ & $5,27 \mathrm{~b}$ & $0,49 \mathrm{~b}$ & $46,22 \mathrm{~b}$ & $12,3 \mathrm{a}$ & $57,1 \mathrm{bc}$ & $69,3 \mathrm{bc}$ \\
\hline $\mathrm{CV}$ & 23,18 & 18,54 & 13,1 & 32,2 & 33,6 & 26.6 \\
\hline
\end{tabular}

CV: coeficiente de variación. Para cada variable, letras distintas indican diferencias significativas (Duncan $\mathrm{p}<0,05$ ).

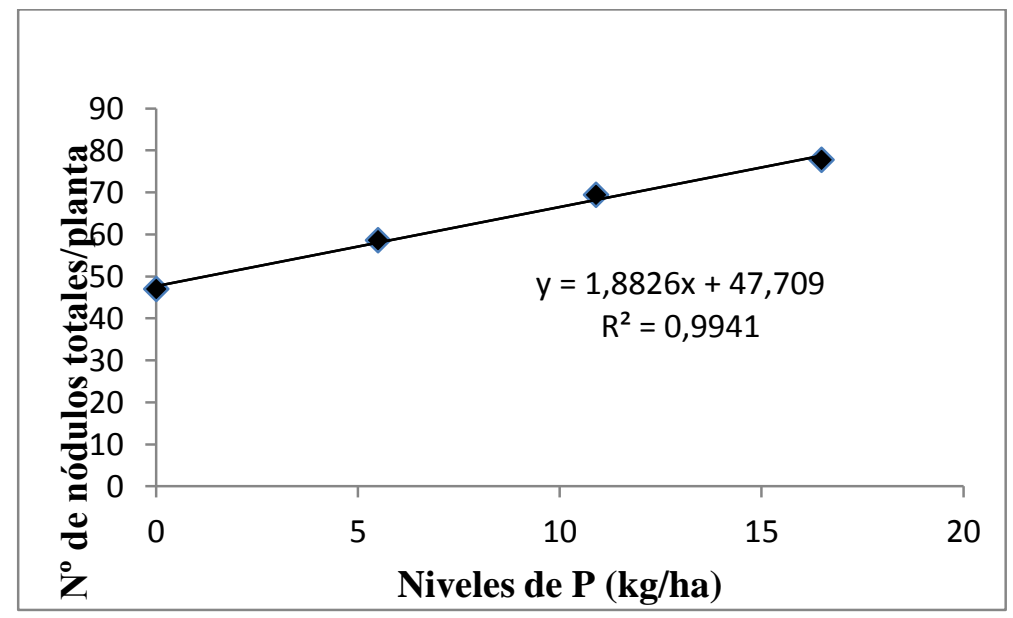

Figura 3. Correlación entre número de nódulos y niveles de $\mathrm{P}(\mathrm{kg} / \mathrm{ha})$ aplicados en los distintos tratamientos. 


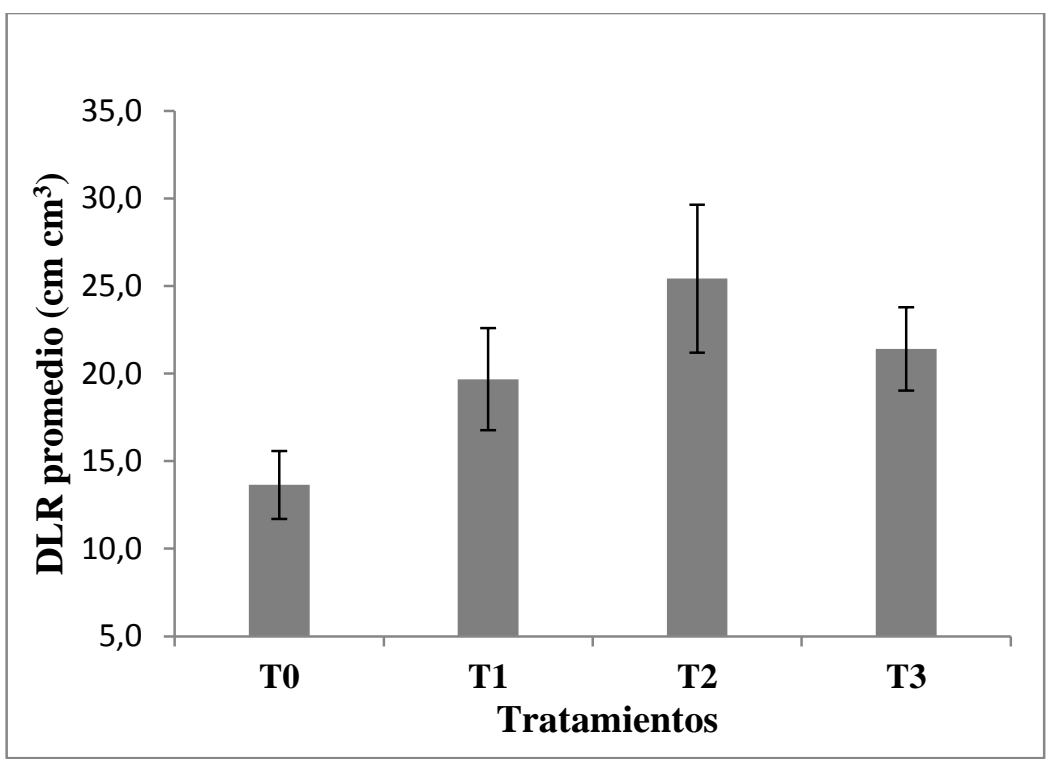

Figura 1. Valores de DLR $\left(\mathrm{cm} \mathrm{cm}^{3}\right)$ promedios. Las barras verticales indican desvíos totales de cada tratamiento

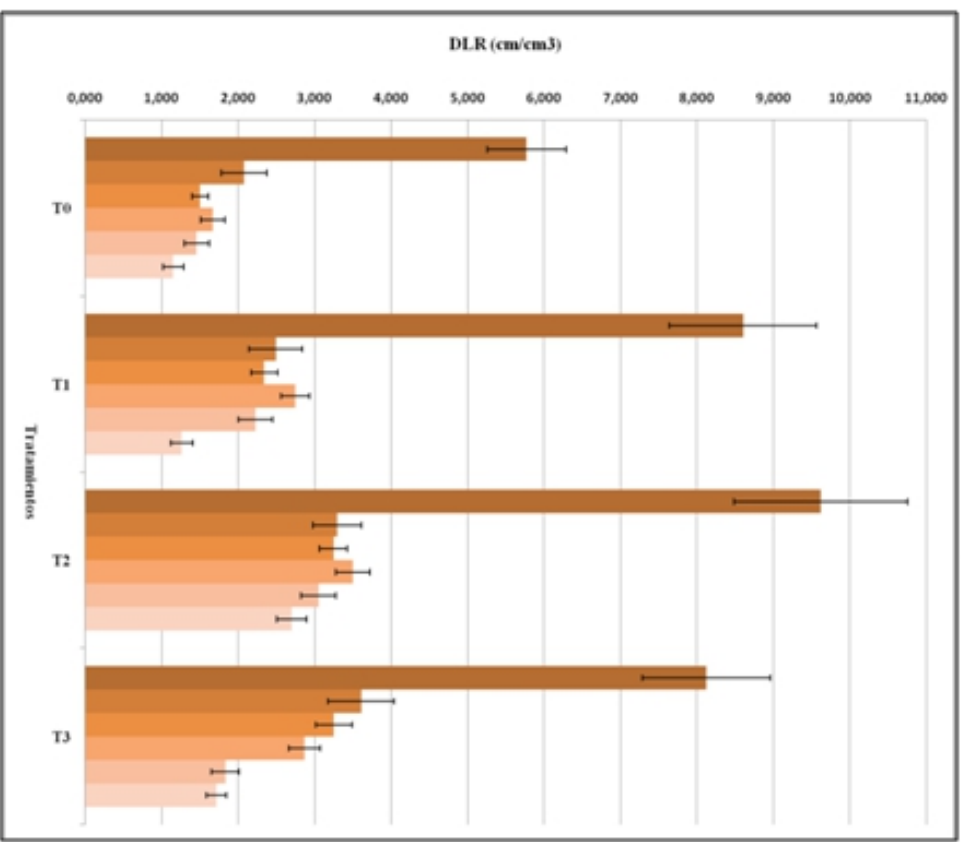

$$
\begin{aligned}
& =0-12 \mathrm{~cm} \\
& =12-24 \mathrm{~cm} \\
& =24-44 \mathrm{~cm} \\
& =44-64 \mathrm{~cm} \\
& =64-84 \mathrm{~cm} \\
& =84-109 \mathrm{~cm}
\end{aligned}
$$

Figura 5: Promedio y desvió estándar de la DLR (cm de raíz/cm3 de suelo) de 0 a $109 \mathrm{~cm}$ de profundidad que fue divido en 6 capas para un análisis más pormenorizado. 


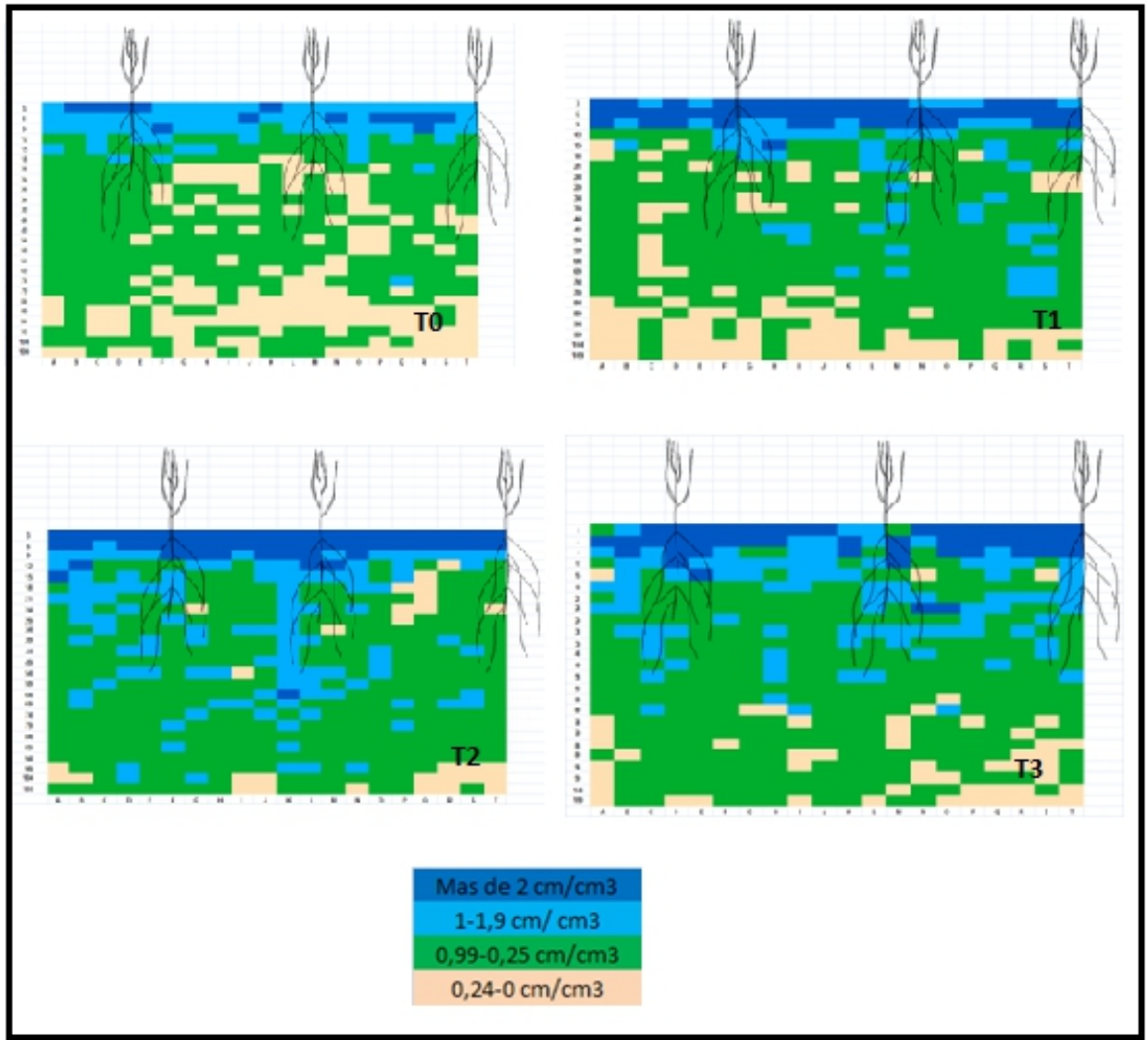

Figura 6. Distribución de DLR en un corte vertical de suelo $(100 \times 109 \mathrm{~cm})$, para cada tratamiento con fertilizante

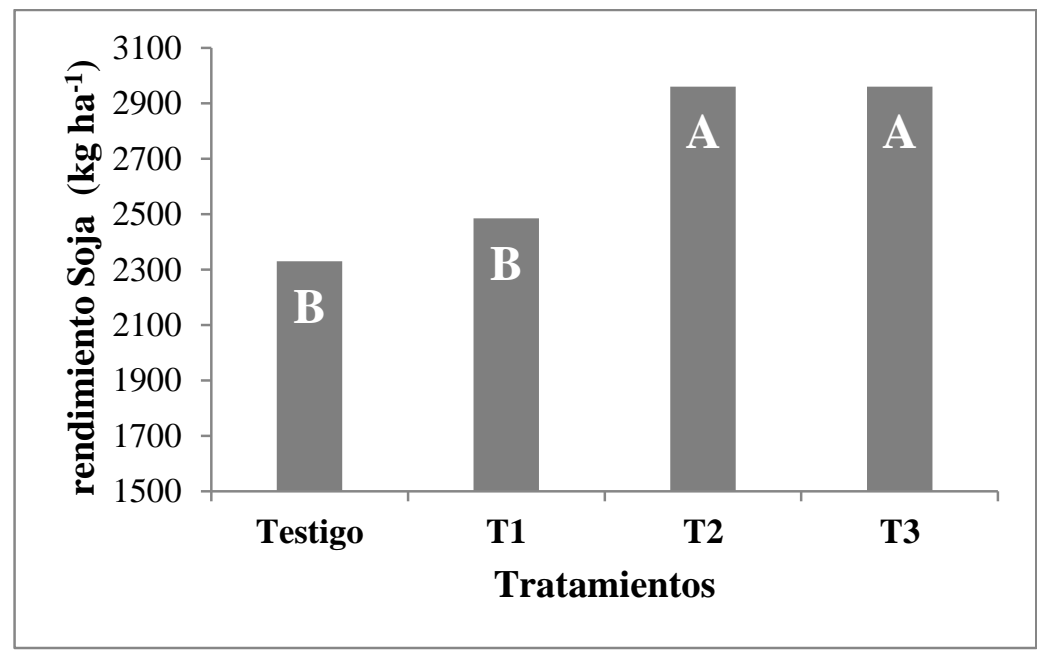

Figura 7: Rendimiento en soja $\left(\mathrm{kg} \mathrm{ha}^{-1}\right)$ en diferentes tratamientos con fertilizante fosforado. Letras distintas indican diferencias significativas según test de Duncan $(\mathrm{p}<0,05)$. 